BNL-NUREG-66831

\title{
FIRE SAFETY IN NUCLEAR POWER PLANTS: A RISK-INFORMED AND PERFORMANCE-BASED APPROACH*
}

\author{
Mohamed A. Azarm and Richard J. Travis \\ Brookhaven National Laboratory \\ Department of Advanced Technology (DAT) \\ Building 475B, P.O. Box 5000 \\ Upton, NY 11973-5000, U.S.A. \\ (516) 344-4922; fax 344-5730; azarm@bnl.gov
}

\begin{abstract}
The consideration of risk in regulatory decision-making has long been a part of NRC's policy and practice. Initially, these considerations were qualitative and were based on risk insights. The early regulations relied on good practices, past insights, and accepted standards. As a result, most NRC regulations were prescriptive and were applied uniformly to all areas within the regulatory scope. Risk technology is changing regulations by prioritizing the areas within regulatory scope based on risk, thereby focusing on the risk-important areas. Performance technology, on the other hand, is changing the regulations by allowing requirements to be adjusted based on the specific performance expected and manifested, rather than a prior prescriptive requirement. Consistent with the objectives of risk-informed and performance-based regulatory requirements, BNL evaluated the feasibility of applying riskand performance-technologies to modifying NRC's current regulations on fire protection for nuclear power plants. ${ }^{(1)}$ This feasibility study entailed several case studies (trial applications). This paper describes the results of two of them. Besides the case studies, the paper discusses an overall evaluation of methodologies for fire-risk analysis to support the risk-informed regulation. It identifies some current shortcomings and proposes some near-term solutions.
\end{abstract}

*The supporting studies for this paper were funded by the U.S. Nuclear Regulatory Commission. The views presented herein are those of the authors and do not reflect NRC's position on the subject.

\section{INTRODUCTION}

Significant advances in, and experiences with, the riskassessment methodology in the past two decades has opened the possibility of a transition to a risk-informed regulatory- and decision-process. A risk-informed approach to decision-making allows the risk insights to be integrated with insights from other disciplines to arrive at a decision whereby public health and safety is explicitly accounted for. The risk-informed process would examine and prioritize the alternatives from a risk perspective. A performance-based approach, on the other hand, is changing the decisionmaking process by allowing requirements to be adjusted based on the specific performance expected and manifested, rather than on the comprehensive prescriptive requirements that are generically applied to all areas. Therefore, it is expected that implementing risk-informed and performance-based decision-making in general, and regulatory practices in specific, would result in more efficient, stable regulatory environment. Towards this end, a series of pilot studies were performed to evaluate the current state of technology and the feasibility of developing risk-informed and performance-based fire-protection requirements. This paper summarizes the results and findings from these studies. Two of these case studies are described: Performance-based fire protection surveillance requirements, and Risk-informed modification to 72-hour criterion to reach cold shutdown. In addition, an overall evaluation of the fire-risk analysis methodologies was conducted. This paper also describes those findings and identifies some of the current shortcomings.

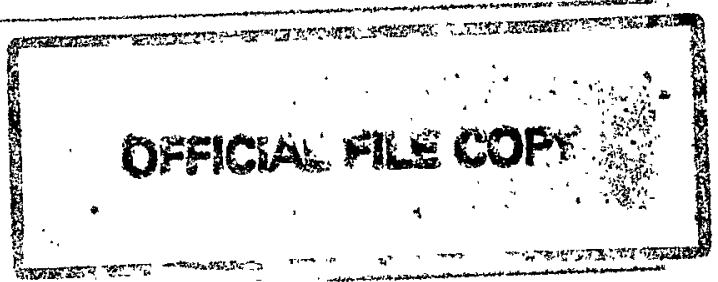


Both the performance-based and risk-informed approach heavily rely on plant data. A separate overall evaluation was made of current plant data to support plantspecific applications of the risk-informed and performancebased requirements. ${ }^{(2)}$ The results indicate that current databases on fire events could support generic fire-risk and performance evaluation, but cannot fully support a plantspecific evaluation. An approach is proposed that uses the findings from plant-specific inspections to tailor the generic evaluation to plant-specific issues. The paper does not propose any specific process for implementing riskinformed and performance-based fire- protection requirements. Instead, it discusses the current methodologies and their potential limitations to determine the proper scope of applications using current state-of-art technology for such requirements.

\section{SURVEILLANCE CASE STUDY}

Surveillance intervals used in nuclear power plants are similar to those specified in the relevant National Fire Protection Association (NFPA) consensus guidelines or standards which are arrived at by groups of experts, based on their experience. Surveillance of fire-protection equipment and installations is specified to occur frequently enough so that the equipment is likely to be in operable condition when the need arises (an accidental fire). The impact of surveillance frequency on the performance (reliability) of standby components has been the subject of many reliability analyses (NUREG/CR-5775). ${ }^{(3)}$ In a performance-based approach, the change in the equipment's reliability or performance due to a change in the surveillance interval is predicted through standard reliability techniques. The resulting reliabilities are used in a riskinformed model (PRAs) to assess surveillance intervals that are more relaxed but, at the same time, have a marginal impact on safety. However, this approach requires engineering information on the types and the extent of the faults detectable by the surveillance (its effectiveness), and the probabilities or the failure rates associated with the occurrence of such faults.

A somewhat different surveillance issue related to the emergency lights was the subject of this study. There are emergency lighting units with at least an 8-hour batterypower supply in U.S. plants in all areas needed to operate safe shutdown equipment, and in the access and egress routes thereto.

Emergency lights are tested annually for a full 8-hour rating. Many of these lights (about 30 to 50 percent) typically fail during the test after 6 to 8 hours. The dominant mechanism is reported to be the depletion of the battery. The fraction of failures and, therefore, the cost for replacing and testing them, can be significantly reduced if the duration of the test is reduced from 8 to 5 hours. Certain types of batteries, such as lead-calcium batteries, are known to have a much shorter lifetime if they are frequently discharged. The rated durations of all types of batteries are a strong function of temperature. The rated duration or the rated capacity is defined by the amperehour capacity assigned to a lead storage cell by its manufacturer for a given discharge time, at a specified electrolyte temperature (typically $25^{\circ} \mathrm{C}$ ) and a specific gravity, to a given end-of-discharge voltage. The batteries usually are designed with some safety margin (about 25 percent); that is, an 8-hour-rated battery, when equalized and new, may have a mean discharge time of about 10 hours. However, when the battery goes through a full discharge, the rated capacity will drop proportionally to the number of discharges.

A probabilistic model and computer code was developed to simulate the reliability of emergency lights that have $X$-hour rated batteries, and are required to operate for $Y$-hours under an emergency, and are periodically endurance-tested for $Z$-hours (where $X, Y$, and $Z$ can be variables). The computer code also can track and evaluate the effect of temperature on the estimated reliability. Therefore, it can differentiate between the reliability of outdoor lights exposed to varying temperatures vs. that of indoor lights in a controlled environment. Using typical values for model parameters, the reliability of emergency lights were evaluated under many different cases and surveillance strategies. Figure-1 is an example of the results generated by the code on the reliability of emergency lights to operate for 8 hours when tested for 8 hours annually. The ability of the method to estimate the failure probability for an emergency light based on its surveillance history and design specifications would be useful in risk-informed and performance-based evaluation. Furthermore, the results support the idea of reducing the duration of the endurance test but leaving the test interval unchanged. The study shows such a modification in surveillance strategy would have a marginal impact on risk, and could avoid the unnecessary cost of replacement.

\section{CASE STUDY ON 72 HOURS TO COLD SHUTDOWN}

Nuclear power plants in the United States are required to achieve cold shutdown within 72 hours after a fire. This implies that for every fire-area, plants have an undamaged train of cold shutdown systems or that any fire damage can be repaired quickly. The LaSalle plant is a typical example. In the LaSalle probabilistic risk assessment (PRA) ${ }^{(4)}$ 


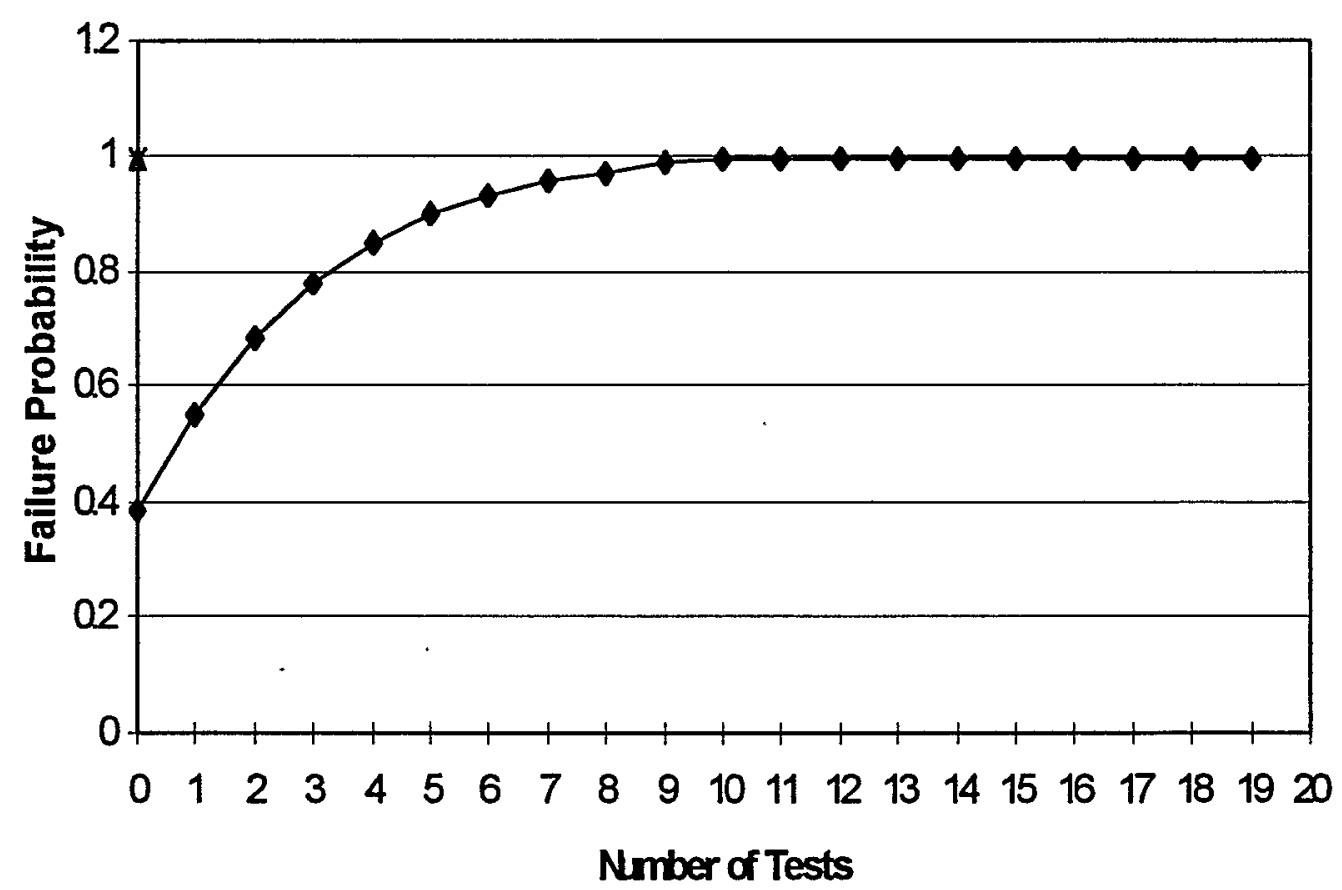

Figure-1: Reliability of battery-operated emergency lights as a function of number of full discharge tests

(NUREG/CR-4832, Vol. 9), no fire- areas contain both RHR (Residual Heat Removal) trains. Additional random failures of the undamaged RHR train generally are required to cause core damage.

To illustrate the approach, the LaSalle PRA analysis of fire-area $A C$ (an area located in the auxiliary building) was used as a surrogate. Fire-area $A C$ is the cable shaft room adjacent to the Unit 2, Division 2 essential switchgear room, and is located in the auxiliary building. This case study postulates that fire-area $A C$ contains equipment associated with both trains of RHR. The postulated damage in fire-area $A C$ is extensive, and it will take more than 72 hours to restore one RHR train. One option is to remove one RHR train from area AC, or to protect it. The alternative option is to allow the plant to reach cold shutdown in a longer period, so that damaged cables could be repaired.

The fire analysis is adapted directly from the LaSalle fire PRA. The probability of a significant fire in fire-area AC can be represented by

$$
\phi_{A C}=\lambda_{A U X} f_{A A C} Q f_{A C} f_{S}
$$

where

$\Phi_{A C}=$ probability of a significant fire in fire-area AC

$\lambda_{A \cup X}=$ auxiliary building's fire frequency

$f_{A A C}=$ area ratio of fire-area $A C$ to that of the auxiliary building

$Q \quad=$ probability that the fire will not be manually suppressed before the critical fire-induced damage occurs

$f_{A C}=$ area ratio within fire-area $A C$ where a significant fire can damage the critical components

$f_{s}=$ severity ratio for a significant fire

The LaSalle fire-modeling determined that a small fire anywhere in fire-area $A C$ can propagate and cause all critical cabling to fail. Therefore, the room-specific area $\left(f_{A C}\right)$ and severity ratio $\left(f_{S}\right)$ terms are 1.0.

Similarly, very little credit can be taken for manual firesuppression because of the comparatively short time before critical damage occurs. A large fire in this area would result in loss of both RHR trains, neither of which could be repaired within 72 hours; this could lead to an accident scenario that eventually can end up in core damage. 
Therefore, the Core Damage Frequency (CDF) contribution of this scenario was estimated to be 5.4E-7 per year (i.e. the value estimated for $\phi_{A C}$ in Equation-1).

The altemative approach assumes that fire-area $A C$ continues to contain cables and equipment for RHR trains $A$ and $B$. A fire is postulated that could extensively damage both RHR trains. The damage cannot be repaired in time to restore safe shutdown capability within $\mathbf{7 2}$ hours; it will take about $\mathbf{2 0 0}$ hours to repair one RHR train. Operating the PCS (Power Conversion System) to cool the core while the extended repair is taking place was considered as an alternative approach. To illustrate it, the LaSalle PRA analysis of fire area AC again is used as a surrogate. For this evaluation additional fire-induced failures were postulated to disable both cold shutdown trains.

From a PRA perspective, the alternative approach presents two modeling challenges. First, the success endstates for the event trees in current PRAs represent cases where primary inventory and core cooling are maintained typically for about 24 hours. This is not necessarily cold shutdown. The assumption is that if core damage can be avoided for this period, a quasi-stable configuration is reached. The alternative approach considers much longer mission times, based on plant-specific considerations and accident sequences. One successful end-state is cold shutdown after repairing one RHR train. This process is estimated to require 200 hours to reach cold shutdown. An alternative success path considers longer-term operation of the power conversion system (PCS), resulting in stable shutdown at 400 hours. Second, many altemative approaches can be expected to emphasize the operator's action. In this instance, the operator's actions to reestablish the condenser and to recover one train of RHR are critical issues. To accurately estimate the likelihood of success and to minimize uncertainty, detailed plant- specific humanreliability analyses are required. For this case study, however, conservative estimates of failure were used for these restorations.

To assess this altemative, the typical PRA must be reevaluated and extended to accurately capture potential systems interactions and important operator's actions. This case study used simplifying assumptions and focused on the long-term PCS operations. A plant-specific analysis is necessary to examine the plant's capability, system interlocks, procedures, and operators' actions. Furthermore, this study examined the alternative of reestablishing the condenser for long-term removal of decay-heat to allow sufficient time to repair one train of RHR shutdown cooling. In accordance with the definition of stable shutdown, longterm operation of the PCS or continued RPV ( Reactor
Pressure Vessel) injection after containment failure are also considered successes.

This case study demonstrated the feasibility of using mean $\triangle C D F$ as a tool towards evaluating the safety equivalence of an altemative approach to a prescriptive requirement. Other considerations include the assumptions, modeling limitations, and sources of uncertainty associated with key events for both the prescriptive-compliance case and the altemative approach. These cautions notwithstanding, a probabilistic approach provides a consistent framework in which to identify key issues, examine sensitivities, and evaluate the safety-equivalence of an alternative approach to a prescriptive requirement.

\section{LIMITATIONS OF CURRENT PRA METHODOLOGIES}

Implementation of risk-informed and performancebased approaches to fire safety at nuclear power plants relies heavily on the fire probabilistic risk assessment (PRA). A fire PRA uses the intemal event PRA models and data, and also includes three additional analyses modules: 1) Fire Data Analysis, 2) Fire Propagation and Suppression Modeling, and 3) Fire Impact Evaluation. The following issues were identified for each of the three modules based on a comprehensive review of current state-of-art fire PRA methodologies.

\section{Fire-Related Data Issues}

Initiating Fire Frequency. The sources of initiating fires in nuclear power plants vary: cable fire, oil fire, transient fire, and cabinet fire. Experience from past fires show large variability in a fire's heat-release rate even for the same type of source. Therefore, it would be important to define what is meant by the initiating fire. What is a minimum size of fire which would be considered challenging enough to be counted for estimating fire frequency and modeling a fire PRA. The minimum size of challenging fire depends on the scope and level of detail in fire PRA models. The initiating size of the fire should define the initial conditions upon which the fire PRA modeling is built. If the fire PRA models include the top level fire-protection features, such as barriers, automatic suppression, and manual fire-fighting then the challenging fires should be defined as those fires that have defeated all lower level protection features, such as combustible control, fire watch, and electrical protection by breakers and fuses. The frequency of challenging fires then could be estimated by consulting the current fire-event databases. ${ }^{(5-9)}$ There are some issues relating to the consistency of databases; however, this topics was addressed previously. ${ }^{(2)}$ 
Equipment-based initiating frequency estimated for challenging fires will be generic ones, and will not address the plant-specific protection features and activities. These lower level fire-protection activities and features are monitored by the plant inspection, and by performancebased monitoring. They are the main reason that precursors to challenging fires do not become major fires. A systematic approach using fire-scenario event trees based on low level fire-protection designs and activities was introduced in reference 2. This approach could be used to incorporate the inspection findings and performance information for a plant-specific estimation of the initiating fire frequencies.

Reliability, Response, and Effectiveness of Detection and Suppression. Automatic detection and suppression systems have been backfitted in nuclear power plants, but, in some cases, automatic fire-detectors and suppression heads may be obstructed by structures, such as cable trays, piping, and ducts. The effect of obstructions on the effectiveness of these automatic features is a source of uncertainty.

The reliability and effectiveness of automatic detectionand suppression-systems should be differentiated. Reliability basically deals with the probability that the component is not failed, whereas effectiveness deals with the probability that the component successfully performs its expected function. The above issue relates to effectiveness of the automatic fire-detection and suppression systems. The response time of fire detectors is affected by the existence of the obstructions. Since fires typically grow exponentially, the slower the detector's response-time, the larger would be the size of the fire at the time of detection; so early detection is very important. It would be important to examine the capability of current codes to estimate the timing of the detector's response.

The case of obstructions higher in the compartment, such as a cable tray near the ceiling, may have less impact since additional mixing with surrounding air would take place higher up. It should be noted that none of these effects, obstructions in the plume, hot layer, or ceiling jets can be addressed by zone models alone.

The suppression system's effectiveness would be impacted by the water droplets hitting an obstruction and not bouncing off, leaving a hole in the spray pattern. If more than one sprinkler were activated, the hole in the spray pattern might be filled in somewhat. It is well known that sprinklers cannot put out a fire that is burning below a low barrier.
Capturing all of the above information in the data estimation is a difficult task. Plant-specific walk downs made as a part of fire PRA activities should focus on identifying the above vulnerabilities.

\section{Fire-Modeling Issues}

Heat-Release Rate. The heat-release rate of a fire source is a complex physical phenomenon, and given the current state-of-the-art modeling techniques, large uncertainties are expected to be associated with the code's prediction. Typically, a simplified bounding estimation using a surface-controlled burning-rate model has been utilized in computer codes, such as COMPBRN, ${ }^{(10)}$ to ensure a conservative estimation of the fire impact. Because of the conservatism of such modeling, there may be cases where the heat-release rate is significantly overpredicted. In these cases, the peak heat-release rates and the associated ranges of variation (uncertainty) may be subjectively determined in light of past occurrences of fire or fire tests and used as input to the code.

Plume Model. The plume algorithm in the COMPBRN fire model is based upon the work done by Zukowski, in which the mass flux into the plume depends on its height above the fire and the burner's diameter. The heat-release rate of the fire is excluded as a factor. This is a different approach and will yield different results $(-20 \%)$ than those obtained from the McCaffrey plume-algorithm used in CFAST, ${ }^{(11)}$ where the calculation of mass flux is based on heat-release rate and its height above the fire. The burner's diameter is excluded as a variable in calculating plume entrainment in the McCaffrey algorithm. Measurements have shown that in a high atrium or large aircrafthanger its omission can cause errors of a factor of 2; but these are areas that are about 60 meters high. For typical areas in nuclear power plants we expect the errors to be significantly less than $50 \%$. The uncertainties associated with semi-empirical plume models and with the variability of basic model parameters are not as significant as the uncertainties associated with the source's heat-release rate.

Model for Compartment(s). Another source of uncertainty is related to the number of compartments analyzed by the model and the compartmental geometry used in the experimental validation. The COMPBRN code is a single-room model that assumes a small (preflashover) fire in a large compartment, where CFAST is a multiroom model (15 max), based on experiments conducted in small rooms. Currently, fire PRAs do not consider fire propagation across fire-rated structural barriers and seals. PRAs assume that probability of such events are negligible, 
considering the large size of, and the slow buming materials (cables) in, the compartments of nuclear power plants. PRA analysts sometimes consider the transport of heat and smoke across compartments as a result of damper failures, especially if smoke-sensitive equipment is in the adjacent room.

Effects of Ventilation. Another limitation and reason for uncertainty is the effects of mechanical ventilation. Most fire-models cannot accurately predict the effects of mechanical ventilation on fire development and the corresponding effects on the fire compartment(s) and contents. Both CFAST and COMPBRN have this feature, but experimental validation is lacking. In contrast to COMPBRN, where vent flow is calculated using empirical equations, CFAST uses Bernoulli's solution for the velocity equation. This solution is augmented for restricted opening by an empirically based flow-coefficient. Forced ventilation is treated as constant flow-rate in COMPBRN, whereas in CFAST, the forced-ventilation mass-flow rate varies with the square root of the pressure drop. Nuclear power plants in the United States are typically multi-room, windowless structures of various sizes and are provided exclusively with forced-ventilation systems. Neither COMPBRN nor CFAST are experimentally validated for such configurations.

Structural Cooling Effect. A considerable cooling effect can come from the masses of cable trays, ventilation ducts, and piping in the upper part of compartments in nuclear power plants. None of the zone models (except, perhaps the French codes) have the ability to calculate the heat transfer by convection from the gas in the hot layer to these structures. So, the gas in the hot layer would actually be much cooler than calculated. If field models were used instead of zone models, predicting the plume, and the upper layers' characteristics would improve dramatically; the processes involving thermal radiation would be more difficult to handle in a field model, however.

\section{Issues in Evaluating the Fire's Impact}

Analysis of Electrical Faults. The effect of fire on electrical (Power, Control, and Instrumentation) cables and cabinets and the ensuing system faults could vary significantly, depending on electrical design. This could cause equipment to either trip if running, or spuriously actuate if in standby. The fault could propagate either directly because of fire propagation, or indirectly through an electrical connection or common enclosure. This issue is known as associated circuit and has been a major part of a fire-impact evaluation. Currently, there no procedural guide for performing such analysis in support of a fire PRA; therefore, there are wide discrepancies among the various fire PRAs reviewed. Currently, the DOE report on Reactor Core Protection Evaluation Methodology ${ }^{(12)}$ (RCPEM) and the IAEA guide ${ }^{(13)}$ for fire-risk analysis are the two documents that could provide such guidance.

Threshold for Equipment-Damage Criteria. Failures of equipment exposed to the harsh environment of a fire and its subsequent suppression are typically determined by a threshold value of an appropriate parameter. This threshold value is referred to as the equipment damageability criteria. As an example, a threshold surfacetemperature is usually considered as a damage criterion for cables. Relative humidity and smoke concentration may be suitable criteria for small electric equipment, such as relays.

Establishing damage criteria is a complex process. Equipment exposed to the thermal environment of a fire may fail either temporarily or permanently. For example, an electronic circuit may temporarily fail (not respond or respond incorrectly) when exposed to high temperature; however, it may recover when the temperature drops. The failure criteria for equipment also depends on its function. Thus, a small insulation-leakage current can cause an instrument cable to fail, whereas the same amount of leakage in a power cable could be non-consequential.

Due to the above difficulties, the damage criteria typically used in PRAs are uncertain. This uncertainty would be directly reflected in the fire-PRA results, since the damage criteria are used to determine the time available for successful suppression. Systematic evaluation of damage criteria for various equipment in nuclear power plants could reduce PRA uncertainties.

Effect of Smoke on Equipment. A fire that is started in one zone might propagate smoke to other zones and with it, the potential for additional damage to equipment. Currently, fire PRAs do not comprehensively treat the question of smoke propagation to other areas and its effect on the operability of components. The extent to which the issue is addressed depends on the analyst, and if addressed, it is typically assessed qualitatively. Some preliminary work was done on evaluating the impact on smoke on electrical/electronic circuits. ${ }^{(14,15)}$

The computer code CFAST can calculate the smoke's concentration and its propagation from one room to another. This feature of the code was not studied as a part of this report and will be explored in future. 
The impact of smoke on the equipment has not been systematically studied. Heavy reliance has been placed on isolating the ventilation ducts by closing the dampers. The current general understanding on this issue is described below:

1. Smoke causes corrosion, depending on what is in it, like $\mathrm{HCL}$ from burning PVC insulation; but this process takes time. A little smoke was shown to cause damage days later if the relative humidity is $70 \%$ or higher. Navy experience demonstrated that affected equipment can be cleaned by a forceful stream of water containing a non-ionic detergent, followed by a rinse with distilled water and drying before corrosion occurs.

2. Smoke also can damage electronic equipment, especially computer boards and power supplies. The extent of the damage may be strongly affected by the force of the fan cooling the electronic equipment which can introduce large amounts of smoke into the housing.

3. Smoke also can impact the operation of relays in the relay cabinet by depositing soot on the contact points. Again, the forced cooling of the relay panel can exacerbate the situation.

Even though some events which have been observed in various industries constitute the bases of the above statements, the authors are not aware of any detailed testing and test results. For the purpose of fire PRAs, some conservative engineering judgments may be made to address the above issues.

Effect of Fire on Human Actions. During a fire some local manual actuations required to bring the plant to safe or stable shutdown may become more difficult since the operator's access to the local areas may be affected by the initiating fire. In addition, the visibility, the availability of emergency lights, and communication may become problematic. Current PRAs could benefit from more attention to these problems.

\section{Uncertainties and Biases}

The issues of uncertainty and evaluation in risk and reliability modeling have been studied more extensively than uncertainties associated with fire modeling. Many fireprotection requirements may be evaluated without the need for fire modeling (e.g., surveillance issues and system issues). For these cases, the issue of uncertainty can be formally addressed and incorporated in the decision-making process. In other cases where evaluating the requirement necessitates using fire modeling, the portion of fire modeling that predicts the fire's heat-release rate was differentiated from the portion that predicts the thermal environment: There are larger uncertainty ranges associated with the predicted heat-release rate than with the thermal environment. The former is the driving force for the plume-mass flow-rate, the ceiling jet's temperature, and finally, the hot-layer temperature which is driven by energy balance. The fire's heat-release rate depends on the initial size of the fire, the growth of fire by propagation and ignition of additional combustibles, and the heat-release rate from them. Current computer codes are judged to perform sound analyses of thermal environment, and some may carry out a formal uncertainty evaluation. On the basis of the case studies performed, the CFAST code performs better in predicting the fire's thermal environment than do other codes. On the other hand, current codes either do not model the source-fire's heat-release rate or do a poor job in predicting it. In any case, knowing the current state-of-the-art, the heat-release rate of the fire source may be best estimated conservatively by using simplified engineering evaluation, subjective judgment, and extrapolating from actual fire events or fire tests.

\section{REFERENCES}

1. M. Dey, M. A. Azarm, R. Travis, et al., "Technical Review of Risk-Informed, Performance-Based Methods for Nuclear Power Plant Fire Protection Analyses," NUREG-1521 Draft report for public comment, July 1998.

2. M. A. Azarm, "Impact of Under-reporting and Event Screening on Fire Frequencies, " Brookhaven National Laboratory, Formal report, JCN W-6276,12/1/97.

3. I. S. Kim, S. Martorell, W. E. Vesely, et al., "Quantitative Evaluation of Surveillance Test Interval Including Test Caused Risks," NUREG/CR-5775, November 1991.

4. J. A. Lambert, D. A. Brosseau, A. C. Payne, et al., "Analysis of the LaSalle Unit 2 Nuclear Power Plant: Risk Method Integration and Evaluation Program (RMIEP) Internal Fire Analysis," Vol. 9, NUREG/CR4832, March 1993.

5. W. Parkinson, G. Solorzano, et al., "Fire Events Database for U.S. Nuclear Power Plants," NSAC-178L, EPRI, June 1992. 
6. Sandia Fire Data Base, "Updated Version of Nuclear Power Plant Fire Data Base, Based on NUREG/CR4586, SAND86-0300 and EPRI-NP-3179." The latest version contains events through December 1989.

7. J. R. Houghton, "A Special Study: Fire EventsFeedback of U.S. Operating Experience," AEOD/S9703, June 1997.

8. "Fire Induced Vulnerability Evaluation (FIVE)," EPRI TR-100370, Final Report, April 1992.

9. W. R. SohIman, "Nuclear Fire Incident Database Query Report," Nuclear Electric Insurance Limited, $3^{\text {rd }}$ International Conference on Fire \& Safety 99, Frankfurt, Germany, February 1999.

10. V. Ho, et al., "COMPBRN Ille: An Interactive Computer Code for Fire Risk Analysis," University of California at Los Angeles, EPRI NP-7283, May 1991.
11. R. D. Peacock, et al., "CFAST, the Consolidated Model of Fire Growth and Smoke Transport," U.S. Department of Commerce, National Institute of Science and Technology, NIST Technical Note 1299, February 1993.

12. "Reactor Core Protection Evaluation Methodology for Fires at RBMK and WER Nuclear Power Plants," U. S. Department of Energy, Revision 1, June 1997.

13. "Treatment of Internal Fires in Probabilistic Safety Assessment for Nuclear Power Plants," Safety report series No. 10, International Atomic Energy Agency, Vienna, 1998.

14. T. J. Tanaka, "Effect of Smoke on Functional Circuits," NUREG/CR-6543, October 1997.

15. T. J. Tanaka, S.P. Nowlen, and D.J. Anderson, "Circuit Bridging of Components by Smoke," NUREG/CR-6476, October 1996. 